\title{
Research on Service Bus Platform Based on New Smart City
}

\author{
Zuocheng Wang ${ }^{1 . a}$, Yanli Luo ${ }^{2 . b}$ and Lixia Xue $\mathrm{X}^{3 . \mathrm{c}}$ \\ ${ }^{1}$ The 38th Research Institute of China Electronics Technology Group Corporation, Hefei, China \\ ${ }^{2}$ Anhui Sun Create electronic CO., LTD. Hefei, China \\ ${ }^{3}$ School computer and information, Hefei University of Technology, Hefei, China \\ a cswangzc@163.com, b442590198@qq.com, ${ }^{\mathrm{c}} 51003239 @$ qq.com,
}

Keywords: new smart city; service bus; multi-source data; cloud computing; big data

\begin{abstract}
With hot construction of smart city, problem such as resources are difficult to share due to information isolated islands, repeated construction, etc. has been exposed. Based on the cloud computing and large data technology, this paper realizes the issues of accessing, processing and storage of multi-source data in urban construction as well as deep analysis and employment of data by service bus platform; also it integrates various kinds of infrastructure services to achieve one procedure from accessing to processing of multi-source data, facilitating the unified application and management, which is the trend of future development of smart city platform construction.
\end{abstract}

\section{Introduction}

Under the situation of hot construction of smart city, some problems have appeared. The eight ministries jointly issue the Guidance of Promoting the Healthy Development of Smart City, clearly pointing out the current four major problems of smart city. The most obvious problem is lack of top-level design and overall planning, which is performed as the chaotic phenomenon: resources are difficult to share across domains due to information isolated islands, for different administration possesses the right of their own smart construction results.

The service bus, combining with the new smart city construction requirements, realizes the functions of resource integration, supervision, application and openness. Also it constructs a sharing, open and safety working environment, which plays a vital role in construction and operation of smart city. The smart city service bus indicates inevitable trend of development of new smart city.

\section{Design of New Smart Service Bus Platform}

The main hierarchical architecture of service bus platform based on the new smart city proposed in this paper is shown in Fig.1.

\subsection{Data source access layer.}

New smart city data is divided into two categories, that is, structured data and unstructured data. Structured data is integrated by basic data from the third party subsystem and platform from the data hub; unstructured data mainly contains videos, pictures and other information. Video network platform integrates accessed front-end equipment of the third-party, storage devices and the third-party platform in line with GB28181 standard, and converts a variety of stream into standard stream.

1) Data hub

Service bus platform achieves issues of high-speed exchange of massive data between 
heterogeneous database and file system to meet the needs of synchronizing cross-system and cross-platform large data. A data sync adapter plug-in, being installed on each server of the third-party data source, is interacted with the adapter plug-in by importing and exporting across the data hub heterogeneous data; data source is connected by reading the parameters of the configuration file; the basic data is preprocessed through data collection, conversion and reading provided by data hub; the basic data is written to the data cache pool through sync and write operation by sync importing and exporting; data batch sync is achieved through the sync strategy.

2) Networking platform

Video network platform docking of service bus platform in accordance with GB / T28181 provides international interface, realizing video resources integration. In this platform, equipment in smart city project is managed, where the size, distribution and detailed information of the equipment are reported to a superior platform; the real-time and the historical video stream of cameras, NVRs, CVRs are given to a superior platform, also the superior platform supervised various front-end control equipment.

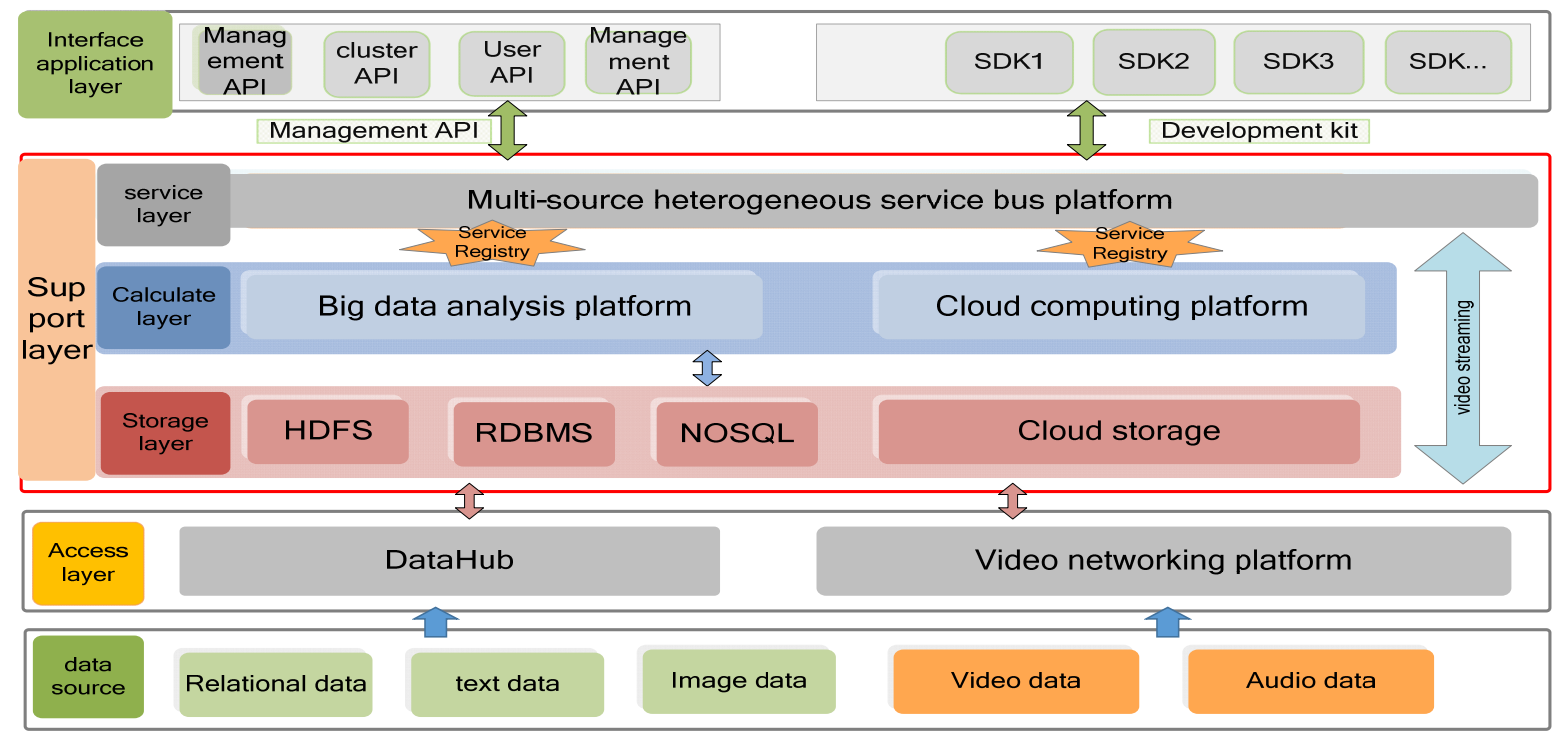

Fig.1. Structure of service bus platform

\subsection{Support platform layer}

The service bus platform, operating storage of the original and value data as well as the analysis and processing of the data through the support platform layer, supporting centralized register, realizes unified internal management and call and the unified external service application.

The support layer mainly includes cloud storage platform, large data analysis platform, cloud computing platform and multi-source heterogeneous service bus platform. The big large data analysis platform, obtaining the structured data from the relational database to carry on the modeling and analysis, excavating the value information, provides multi-source heterogeneous service bus to an upper layer. Cloud computing platform first obtains video streaming from the network platform to analysis. Then the analyzed video clips and pictures are stored to the cloud storage platform. At last the big data analysis platform and cloud storage platform provides relevant data to multi-source heterogeneous service bus.

1) Cloud storage platform

Cloud storage platform, by applying cloud storage technology, cluster technology and video transcoding technology to the enterprise's massive data storage, synchronization and sharing management, applies upload, download, on-demand, search and other functions to video and picture 
resources in billion-grade. Through the expanded the service cluster providing the appropriate basic services vie the basic storage cluster, a common service interface is informed.

2) Big data analysis platform

The big data analysis platform focuses on processing, analysis and display business of massive and heterogeneous data as the core module of service bus platform. It supports data access, data self-help modeling, multidimensional analysis and visualization show, providing data support services for the upper layer. The overall design is shown as Fig 2.

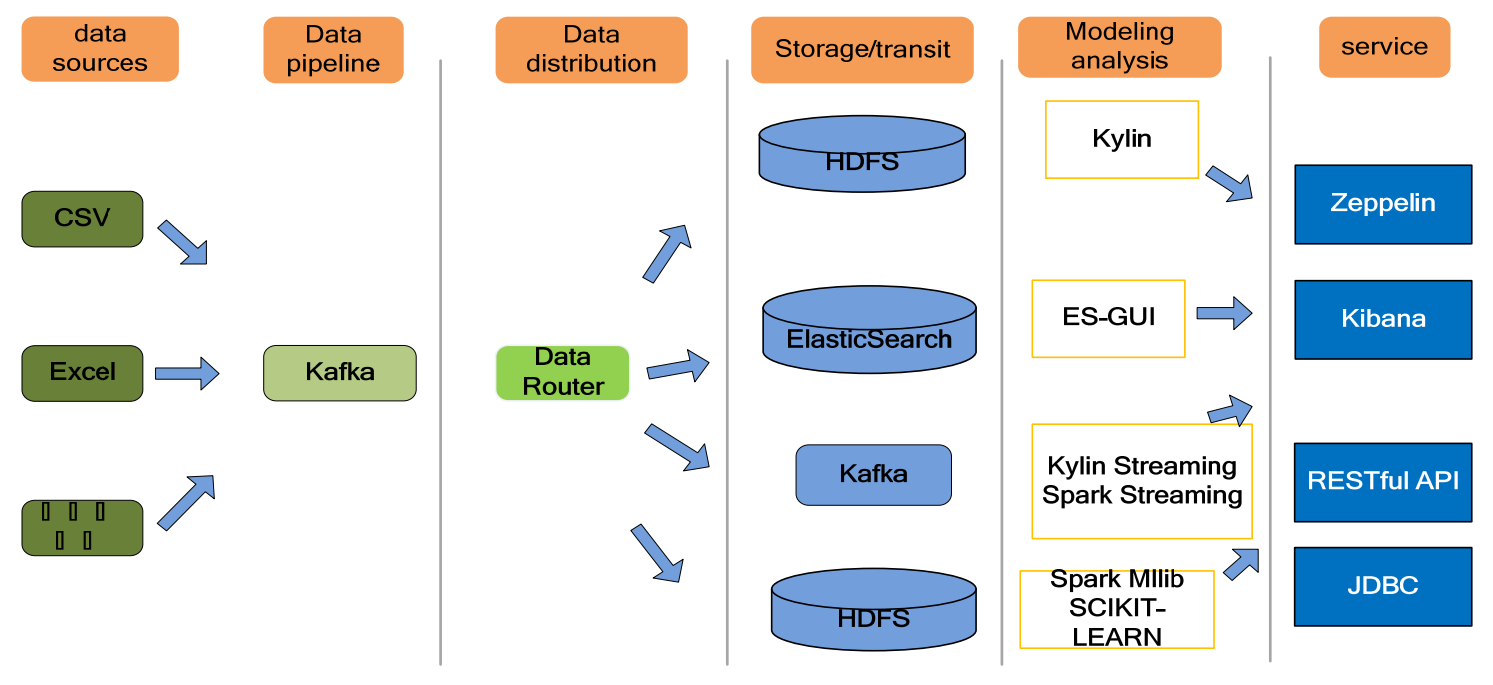

Fig.2. Overall design

3) Cloud computing platform

Cloud computing platform carries visual management and scheduling on a variety of algorithms and application services by using the latest container technology, which achieves the algorithm management on the cloud computing platform, tasks, mirroring, nodes and other management necessities. Cloud computing platform registers task management service, image management service, node management service, algorithm call service, the configuration management service and operation log service on the multi-source heterogeneous service bus in the form of interfaces.

4) Multi-source heterogeneous service bus platform

Multi-source heterogeneous service bus platform provides integration management on the distributed, message-based, standard interface and service, to solve the complex state of communication and data exchange. Multi-source heterogeneous service bus achieves the service registration, discovery, monitoring and management on the lower level, and provides the specified form of service according to the needs of the application layer, so as to complete the unified management of services and services on the unified scheduling.

\subsection{Application layer}

The service bus platform realizes the centralized visual display of each sub-product. Meanwhile, it operates central management on the overall platform security authentication, cloud service management, subsystem management and system configuration.

\section{Summary}

This paper, integrating the construction requirements of the new smart city, introducing the Hadoop big data and the docker container technology, taking the data as the core, basing on the double cloud (cloud computing, cloud storage), studies and realizes the new smart city service bus platform. The platform meets the core needs of resource integration and supervision towards the 
lower level and the application and openness towards the upper level by building a sharing, open and safety running environment, which provides a powerful support for the construction and operation of the new intelligent smart city. It is the inevitable trend and the necessary demand for development of a new type of smart city.

\section{Acknowledgements}

This work is partially supported by the Fundamental Research Funds for the Central Universities of China (JZ2014HGBZ0059).

\section{References}

[1] Liu Yunheng, Liu Yaozong: Hadoop-based Public Security Video Big Data Processing Method [J].Computer Science, 2016, 43(6A):448-451, 475.

[2] Zhang Tong, Lu Aiyu, Mo Jianwen: Design of live video streaming, video-on-demand system based on Darwin [J].Application of Electronic Technique, 2015, 41(8):124-127.

[3] Fan Jing, Xiong Lirong, Xu Cong: Research and application of data integration in distributed enterprise service bus platform [D].Computer Science, 2014, Vol. 41, No.2.

[4] Zhang jian, Xie Tianjun: Based on the Docker platform as a service architecture research [D]. Information technology and information technology, 2015 (10): 131-134.

[5] CHEN Xu-wen, WU Yong-na: Research on Excellent Resource Sharing Model Based on Cloud Storage [J]. Jiangxi Science,2014(2):234-237.

[6] Lin Sui, Huang Jian, Jiang Wenchao, Qin Guomin: A Secure Cloud Storage Model Based on HDFS [J]. Journal of Guangdong University of Technology, 2014(3):49-54.

[7] Song Huazhu, Duan wenjun, Liu xiang: Ontology Storage Model Based on HBase [J].Computer Science, 2016, 43(6):39-43. 